\title{
Effects of adiabatic compression on thermal convection in super-Earths of various sizes
}

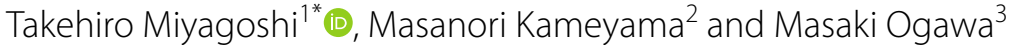

\begin{abstract}
We present two-dimensional numerical models of thermal convection of a compressible fluid in the mantles of super-Earths calculated under the truncated anelastic liquid approximation to discuss how adiabatic compression affects the thermal convection, depending on planetary mass. The convection is driven by basal heating, the viscosity depends on temperature, and the thermal expansivity and the reference density depend on the depth. We varied all of the magnitude of adiabatic heating, the Rayleigh number, the depth profile of the thermal expansivity, and that of the reference density in accordance with the planetary mass. The effects on thermal convection become substantial, when the planetary mass normalized by the Earth's mass $M_{p}$ exceeds a threshold $M_{c^{\prime}}$ about 4 . Hot plumes ascending from the core-mantle boundary become thinner with increasing $M_{p}$; they become almost invisible except around the core-mantle boundary, when $M_{p}>M_{c}$. The lithosphere that develops along the surface boundary due to the temperature dependence of viscosity becomes thicker with increasing $M_{p}$ and is about twice as thick as that at $M_{p}=1$ when $M_{p}=9.4$. The convective velocity is almost independent of $M_{p}$. These results are in a striking contrast with earlier predictions that are made based on the models where the effects of adiabatic compression are neglected; it is important to take account of the effects of adiabatic compression properly in the exploration of mantle dynamics such as plate tectonics and hot spot volcanisms in massive super-Earths. Further researches are necessary to clarify the dependence of $M_{c}$ on the surface temperature and the material properties of the convecting mantle.
\end{abstract}

Keywords: Mantle convection, Super-Earths of various sizes, Adiabatic compression, Numerical simulations

\section{Introduction}

Motivated by recent detection of a large number of super-Earths, i.e., extrasolar planets with a mass of up to ten times the Earth's (e.g., Borucki et al. 2011), many researchers have explored dynamics of the mantle of super-Earths, because it is a key to understanding their tectonics, thermal history, and surface environments (e.g., Valencia et al. 2007; Valencia and O'Connell 2009; van Heck and Tackley 2011; Foley et al. 2012; Lenardic and Crowley 2012; Stein et al. 2013; Tackley et al. 2013; Stamenković and Breuer 2014). In the terrestrial planets of our solar system, two of the most important factors

\footnotetext{
*Correspondence: miyagoshi@jamstec.go.jp

${ }^{1}$ Department of Deep Earth Structure and Dynamics Research, Japan Agency for Marine-Earth Science and Technology, 3173-25 Showa-machi, Kanazawa-ku, Yokohama, Kanagawa 236-0001, Japan

Full list of author information is available at the end of the article
}

that dominate mantle dynamics are the lithosphere and hot ascending plumes (e.g., Schubert et al. 2001; Davies 2011). Whether the lithosphere on super-Earths is rifted into mobile plates as observed on the Earth or remains stagnant as observed on Venus and Mars has been a central issue, and the predictions made in the literature range from the stagnant lithosphere (e.g., O'Neill and Lenardic 2007; Stein et al. 2013) to active plate tectonics (e.g., Valencia et al. 2007; Valencia and O'Connell 2009; van Heck and Tackley 2011; Foley et al. 2012; Tackley et al. 2013). In their review of parameterized convection models for super-Earths, Stamenković and Breuer (2014) conclude that whether plate tectonics can operate or not sensitively depends on the detail of the parameterization. The vigor of hot ascending plumes, although it has not drawn so much attention, is also an important issue, since hot plumes affect the activity of hot spot volcanism and 
large igneous provinces that have been suggested to rift the lithosphere on the Earth (e.g., Richards et al. 1989). Before challenging these issues on the real super-Earths, however, it is important to explore thermal convection in the mantle of super-Earths as a problem of fluid dynamics. Recently, Miyagoshi et al. (2014, 2015, 2017) have focused on the effects of adiabatic compression in superEarths of ten times the Earth's mass, and found that the effects are substantial, as summarized below. Here, we apply our earlier numerical models to super-Earths of various mass to clarify the threshold in planetary mass above which the effects of adiabatic heating we found become important.

An important finding of Miyagoshi et al. $(2014,2015)$ is that adiabatic compression substantially reduces the vigor of thermal convection in a massive super-Earth in contrast to the predictions of many of earlier studies that are based on the Boussinesq approximation where adiabatic compression is neglected (e.g., Valencia et al. 2007; Valencia and O'Connell, 2009; van Heck and Tackley 2011; Foley et al. 2012). In their models, Miyagoshi et al. $(2014,2015)$ found that the lithosphere becomes much thicker and hot ascending plumes become thinner than expected from Boussinesq models in a planet of ten times the Earth's mass. These results suggest that it is necessary to revisit the issue of plate tectonics and hot spot volcanism in super-Earths, taking account of the effects of adiabatic heating (e.g., Valencia et al. 2007; Valencia and O'Connell 2009; van Heck and Tackley 2011; Foley et al. 2012). In this paper, we apply our earlier models to superEarths of various mass $M_{\mathrm{p}}$ (the planetary mass divided by the Earth's mass) $1,2,4,6$, and 9.4 to clarify the minimum planetary mass above which the effects of adiabatic compression on mantle convection become prominent. To highlight the effects of adiabatic compression, we keep our models as simple as possible and neglect other factors like the possible effects of complicated rheology of mantle materials, the process of planetary formation, the boundary condition on the core-mantle boundary, and internal heating.

\section{Model}

We only briefly describe the model and basic equations here; the readers are referred to our previous studies for more detail (Miyagoshi et al. 2014, 2015, 2017).

We calculated a thermal convection of an infinite Prandtl number fluid with a temperature-dependent viscosity in a two-dimensional rectangular box of the aspect ratio four under the truncated anelastic liquid approximation (TALA). All of the boundaries are shear stress free and impermeable. The surface temperature $T_{\mathrm{s}}^{*}$ and the bottom temperature $T_{\mathrm{b}}^{*}$ are both fixed, and the normalized surface temperature $T_{\mathrm{s}}^{*} /\left(T_{\mathrm{b}}^{*}-T_{\mathrm{s}}^{*}\right)$ is fixed at 0.1. (The asterisks stand for dimensional quantities). The sidewalls are insulating.

The employed non-dimensional momentum equation is

$$
\begin{aligned}
& -\nabla p+\nabla \cdot \tau+R a \cdot \bar{\rho}(z) \cdot \alpha(z) \cdot T \cdot \mathbf{e}_{\mathbf{z}}=0, \\
& \tau_{i j}=\eta(T)\left[\left(\nabla \mathbf{u}+{ }^{\mathbf{t}} \nabla \mathbf{u}\right)_{i j}-\frac{2}{3} \nabla \cdot \mathbf{u} \delta_{i j}\right],
\end{aligned}
$$

the mass conservation equation,

$$
\nabla \cdot[\bar{\rho}(z) \mathbf{u}]=0,
$$

and the energy equation

$$
\bar{\rho}(z)\left[\frac{\mathrm{d} T}{\mathrm{~d} t}+D i \cdot \alpha(z) w T\right]=\nabla^{2} T+\frac{D i}{R a} \tau_{i j} \frac{\partial u_{i}}{\partial x_{j}} .
$$

The length scale for the normalization is the mantle depth $d^{*}$; the temperature scale is the temperature difference $\Delta T^{*} \equiv T_{\mathrm{b}}^{*}-T_{\mathrm{s}}^{*}$; the time scale is $d^{* 2} / \kappa^{*}$ where $\kappa^{*}$ is the thermal diffusivity. $\bar{\rho}(z)$ and $\alpha(z)$ are the depth-dependent reference density and thermal expansivity, respectively; $\eta(T)$ is the viscosity; $\delta_{i j}$ is the Kronecker's delta; $z$ is the height measured from the bottom boundary; $p$ is nonhydrostatic pressure; $\mathbf{u}$ and $\mathbf{e}_{\mathbf{z}}$ are the fluid velocity vector and the vertical unit vector; $w$ is the vertical component of fluid velocity; $t$ is time; $R a$ is the Rayleigh number defined by $R a=\rho_{0}^{*} \alpha_{0}^{*} g^{*} \Delta T^{*} d^{* 3} / \eta_{0}^{*} K^{*} ; D i$ is the dissipation number defined by $D i=\alpha_{0}^{*} g^{*} d^{*} / C_{\mathrm{p}}^{*}$. Here, $\rho_{0}^{*}$ and $\alpha_{0}^{*}$ are the density and thermal expansivity at the surface, respectively, $g^{*}$ is the gravity, $C_{\mathrm{p}}^{*}$ is the specific heat, and $\eta_{0}^{*}$ is the viscosity on the bottom boundary that is chosen as the reference viscosity.

The viscosity $\eta$ depends on the temperature as $\eta=\eta^{*} / \eta_{0}^{*} \exp \left[\ln (r)\left(T_{\mathrm{b}}-T\right)\right]$ where $r$ is the viscosity contrast between the top and bottom boundaries; we fixed $r$ at $10^{5}$ regardless of $M_{\mathrm{p}}$, in order to focus on the effects of adiabatic compression. The value of $r$ is chosen so that the lithosphere develops along the cold surface of the planet. We do not take account for the pressure-dependent viscosity, because the physical properties under very highpressure expected in massive super-Earths are still not well known. For example, Karato (2011) suggests that such a high pressure may reduce rather than increase the viscosity. As shown in Table 1, the thermal expansivity decreases with the hydrostatic pressure that is calculated from the depth as

$$
\bar{p}^{*}(z)=\left(127.4 M_{\mathrm{p}}+7.241\right)(1-z) \quad[\mathrm{GPa}]
$$

(Tachinami et al. 2014; Miyagoshi et al. 2014, 2015, 2017). The reference density depends on $z$ as

$$
\bar{\rho}(z)=1+\left[1.75\left(M_{\mathrm{p}}\right)^{0.258}-1\right](1-z) .
$$

The basic equations are discretized by a finite difference method. We employed the ACUTEMAN numerical code (Kameyama 2005; Kameyama et al. 2005) for the 
Table 1 The pressure dependence of the thermal expansivity assumed in the model

\begin{tabular}{ll}
\hline Pressure (GPa) & $\begin{array}{l}\text { Thermal } \\
\text { expansivity } \\
\left(\mathbf{1 0}^{-5} \mathbf{K}^{-1}\right)\end{array}$ \\
\hline 0 & 4.0 \\
10 & 3.5 \\
30 & 2.6 \\
60 & 1.86 \\
100 & 1.38 \\
150 & 1.10 \\
300 & 0.701 \\
600 & 0.3764 \\
1000 & 0.3034 \\
1500 & 0.2587 \\
\hline
\end{tabular}

calculation. The employed mesh is uniform and contains 1024 times 256 grid points.

We carried out numerical calculations for planets with $M_{\mathrm{p}}=1,2,4,6$, and 9.4. The corresponding values of $(R a, D i)$ are $\left(5 \times 10^{7}, 0.87\right),\left(1.3 \times 10^{8}, 1.49\right),\left(3.2 \times 10^{8}\right.$, $2.57),\left(5.5 \times 10^{8}, 3.52\right)$, and $\left(1.0 \times 10^{9}, 5.00\right)$, respectively. In these estimates, we assume that the modeled planet has a similar chemical composition with the Earth's and that $g^{\prime \prime}$ and $d^{*}$ depend on the planetary mass normalized by the Earth's mass $M_{\mathrm{p}}$ as $g^{*}=g_{\oplus}\left(M_{\mathrm{p}}\right)^{0.5}$ and $d^{*}=d_{\oplus}\left(M_{\mathrm{p}}\right)^{0.28}$, respectively (Valencia et al. 2006, 2007), where the subscript $\oplus$ stands for the Earth's value; $R a$ thus calculated depends on $M_{\mathrm{p}}$ as $R a=R a_{\oplus}\left(M_{\mathrm{p}}\right)^{1.34}$, while $D i$ depends on $M_{\mathrm{p}}$ as $D i=D i_{\oplus}\left(M_{\mathrm{p}}\right)^{0.78}$. Here, $D i_{\oplus}=0.87$ and $R a_{\oplus}=5 \times 10^{7}$, as estimated with $C_{\mathrm{p}}=1.3 \times 10^{3} \mathrm{~J} / \mathrm{kg} / \mathrm{K}, \alpha_{0}=4 \times 10^{-5} \mathrm{~K}^{-1}, g_{\oplus}=9.8 \mathrm{~m} /$ $\mathrm{s}^{2}$, and $d_{\oplus}=2900 \mathrm{~km}$. We fixed the temperature contrast across the mantle $\Delta T^{*}$ at $3000 \mathrm{~K}$ in the estimates of $R a$; the effects of variation in $R a$ due to a variation in $\Delta T^{*}$ on numerical results can be readily estimated from the parametrized relationships presented in Tachinami et al. (2014) and Miyagoshi et al. (2015). We also fixed the nondimensional surface temperature $T_{\mathrm{s}}$ at 0.1 , since Tachinami et al. (2014) has already investigated its effects on numerical results for iso-viscous models.

We shortly note the validity of TALA in the numerical model of mantle convection in the presence of strong compression. Compared with its untruncated counterpart (ALA), the effect on buoyancy of dynamic pressure is neglected in TALA. Such a truncation can be safely done in the mantles of terrestrial planets particularly with large mass, because the dynamic pressure is much smaller than the static one in the planetary interiors. This has been already confirmed from numerical results with $M_{\mathrm{p}}=10$ in Kameyama and Yamamoto (2018).

\section{Results}

The left column of Fig. 1 shows snapshots of the distribution of potential temperature $T_{\mathrm{p}}(x, z)$ (color) and fluid velocity (arrows) we obtained at various $M_{\mathrm{p}}$ at the statistically steady state, while the right column shows the horizontal average $\overline{T_{\mathrm{p}}}$ of the potential temperature $T_{\mathrm{p}}(x, z)$. The potential temperature is calculated from the temperature $T(x, z)$ as shown in Fig. 2

$$
T_{\mathrm{p}}(x, z)=T(x, z) \exp \left(-D i \int_{z}^{1} \alpha(\xi) d \xi\right),
$$

(Miyagoshi et al. 2014, 2015, 2017). We present $T_{\mathrm{p}}$ rather than $T$ in Fig. 1, because $T_{\mathrm{p}}$ is more directly related to the buoyancy force that drives thermal convection.

At $M_{\mathrm{p}}=1$, hot plumes ascending from the core-mantle boundary are as prominent as the cold plumes that descend from the top cold thermal boundary layer (TBL), and the convective velocity around the cold plumes is comparable to that around the hot plumes. The heads of the hot plumes reach the base of the top TBL.

Hot ascending plumes become, however, less prominent as $M_{\mathrm{p}}$ increases: At $M_{\mathrm{p}}=2$, the convective flow induced by hot plumes is still an important element of the convection in the mantle. The excess temperature of the heads of hot plumes with respect to the surrounding mantle is, however, smaller than that calculated at $M_{\mathrm{p}}=1$ shown in Fig. 1a. At $M_{\mathrm{p}}=4$ and 6, the excess temperature of hot plume heads becomes even smaller. Hot plumes become faint except around the core-mantle boundary, and the heads are often detached from the stems of hot plumes before they ascend to the base of the top TBL. The excess temperature of hot plumes rapidly decreases as they ascend, while cold descending plumes are prominent at all depths. At $M_{\mathrm{p}}=9.4$, hot plumes become almost invisible, while cold descending plumes are still conspicuous. The effects of adiabatic compression on temperature contrast between plumes and the surrounding mantle are more conspicuous for hot plumes than for cold plumes, because the effect is proportional to the temperature (Eq. 4).

Figure 2 shows snapshots of the temperature distribution from which the potential temperature shown in Fig. 1 is calculated. Cold plumes are conspicuous at all $M_{\mathrm{p}}$. Hot plumes, in contrast, become fainter as $M_{\mathrm{p}}$ increases and are almost invisible at $M_{\mathrm{p}}=4$ or larger, although the structures can be slightly observed in the potential temperature distribution.

Figure $3 \mathrm{a}$ shows the normalized thickness of the top TBL, or the lithosphere, plotted against $M_{\mathrm{p}}$. The base of the lithosphere is located at the depth level where $\overline{T_{\mathrm{p}}}-T_{\mathrm{s}}$ becomes $98 \%$ of $\overline{T_{\mathrm{pm}}}-T_{\mathrm{s}}: \overline{T_{p m}}$ is the local maximum of $\overline{T_{\mathrm{p}}}$ in the shallow mantle (Miyagoshi et al. 2015). 

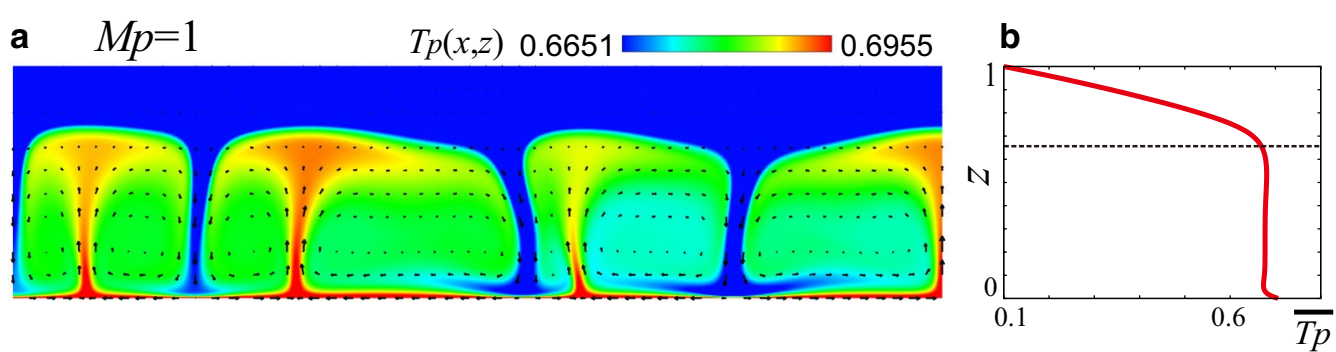

c $M p=2$

$0.5890 \square 0.6194$

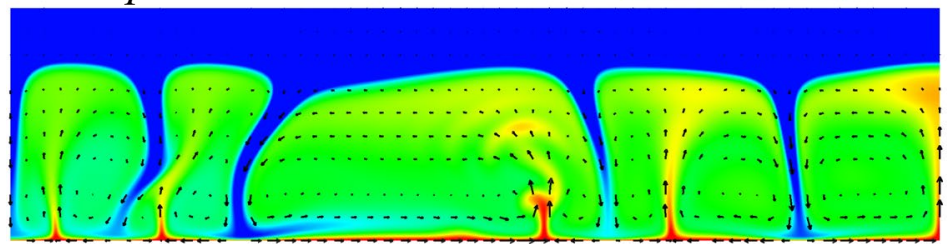

d

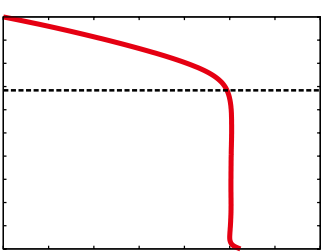

e $M p=4$

$0.5224 \square 0.5528$

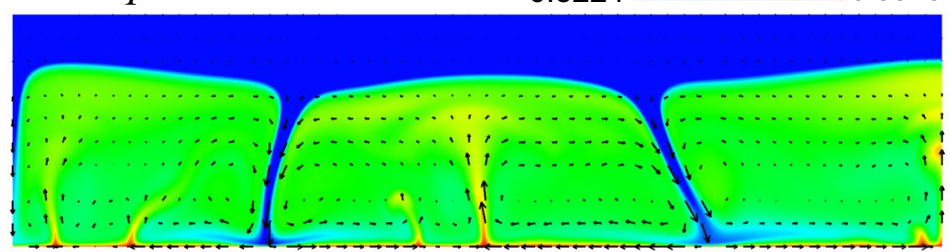

f

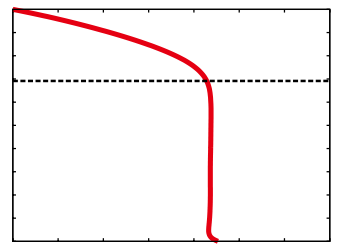

\section{g $M p=6$}

0.4945

0.5249

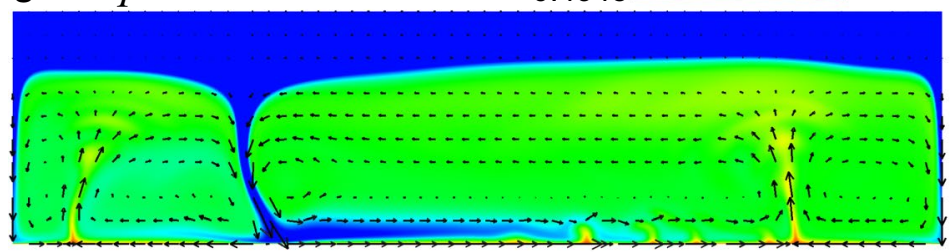

h

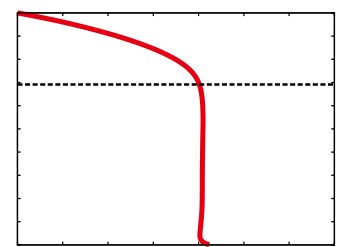

i $\quad M p=9.4$

$0.4732 \square 0.5036$

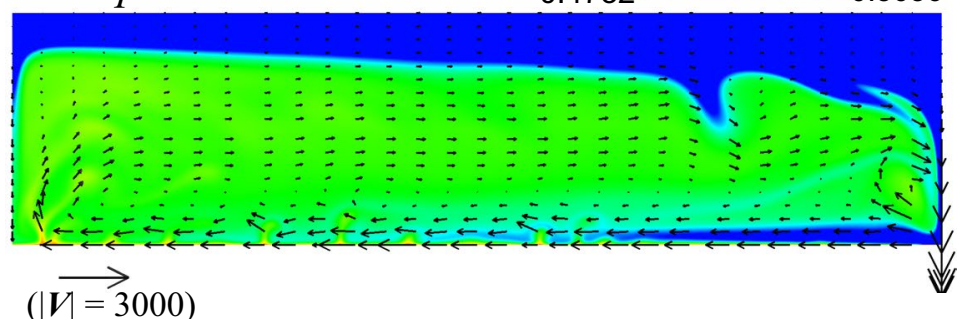

j

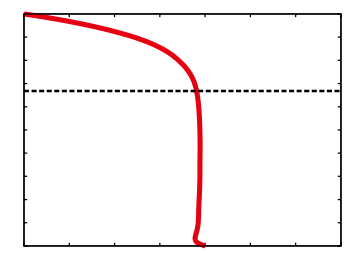

$$
(|\vec{V}|=3000)
$$

Fig. 1 The left column shows snapshots of the distribution of the non-dimensional potential temperature $T_{p}(x, z)$ (color) and fluid velocity (arrows) at various $M_{p} \cdot T_{p}$ is normalized with the temperature unit of $\Delta T^{*}=3000 \mathrm{~K}$. The color scale is changed from panel to panel so that $T_{p}$ of the isothermal core of the convection is represented by green color. See the sample arrow beneath the panel (i) for the non-dimensional magnitude of the velocity. The right column shows the horizontally averaged $T_{p}(x, z), \overline{T_{p}}$ (the red lines). The dashed black lines show the location of the base of the lithosphere (for details, see the text)

The height of the base of the lithosphere thus defined is shown in the right column in Fig. 1 by the black dashed lines. The figure shows that the normalized thickness of the lithosphere is almost independent of $M_{\mathrm{p}}$. This behavior is different from that found in the earlier models where the Boussinesq approximation is employed: Under this approximation, the flux of convective heat transport $q$, which is approximately proportional to the inverse of the normalized thickness of the lithosphere, is known to depend on the Rayleigh number as $q \propto R a^{0.258}$ (e.g., 
a $\quad M p=1$

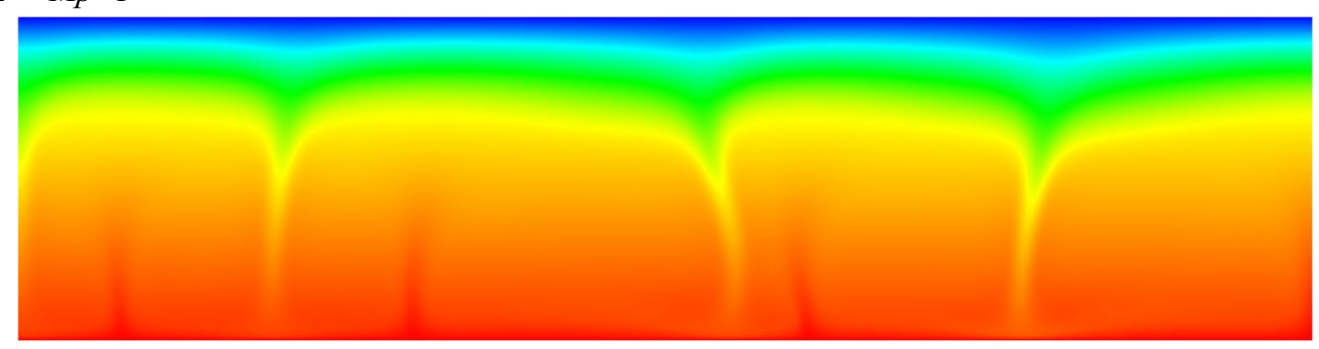

b $M p=2$

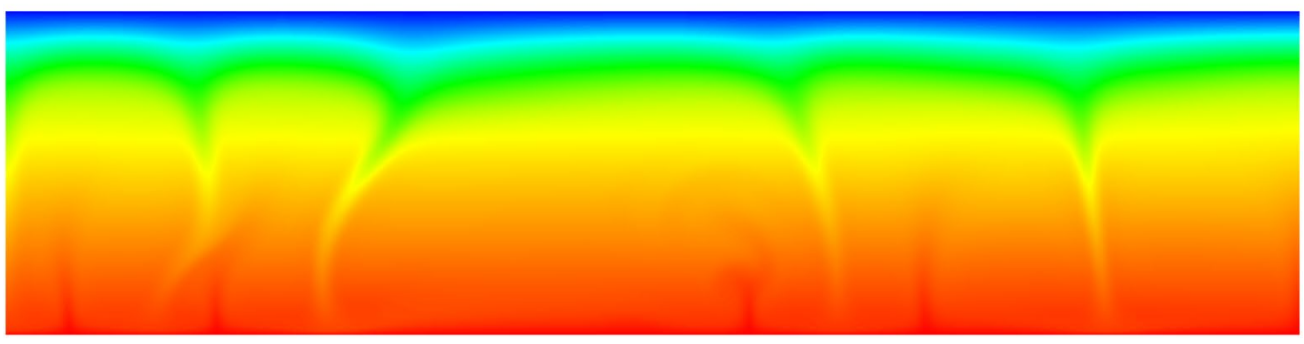

C $M p=4$

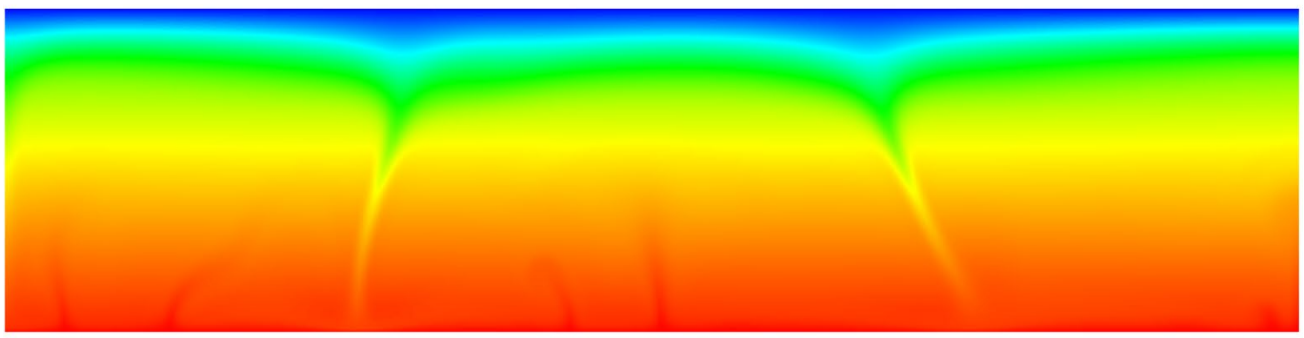

d $M p=6$

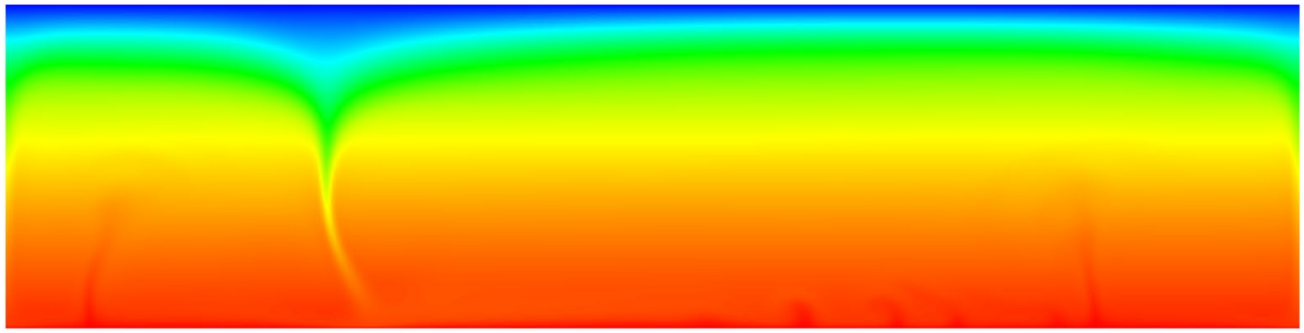

e $\quad M p=9.4$

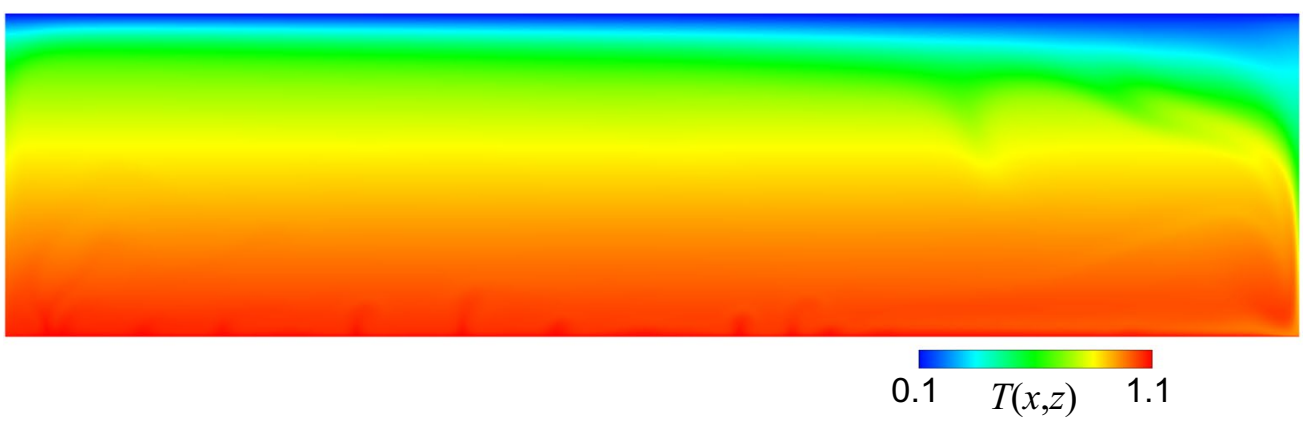

Fig. 2 Snapshots of the temperature distribution (color) from which the potential temperature presented in Fig. 1 is calculated 


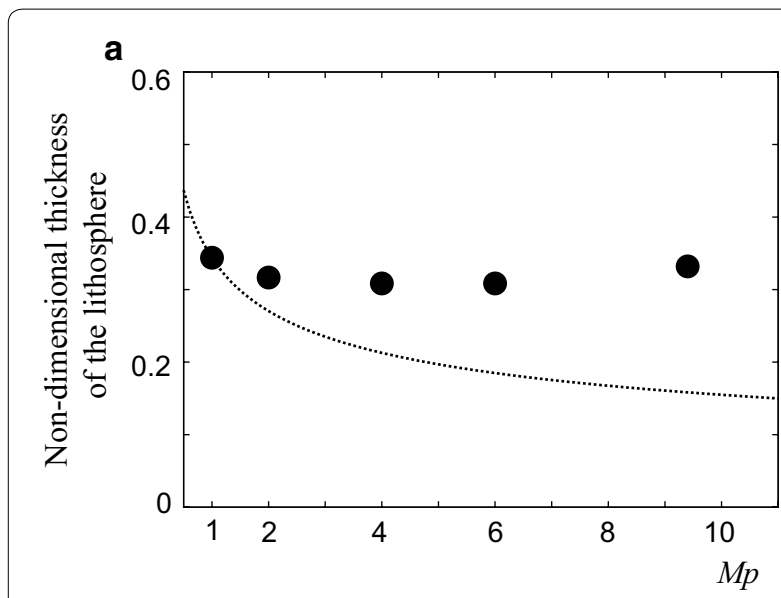

b

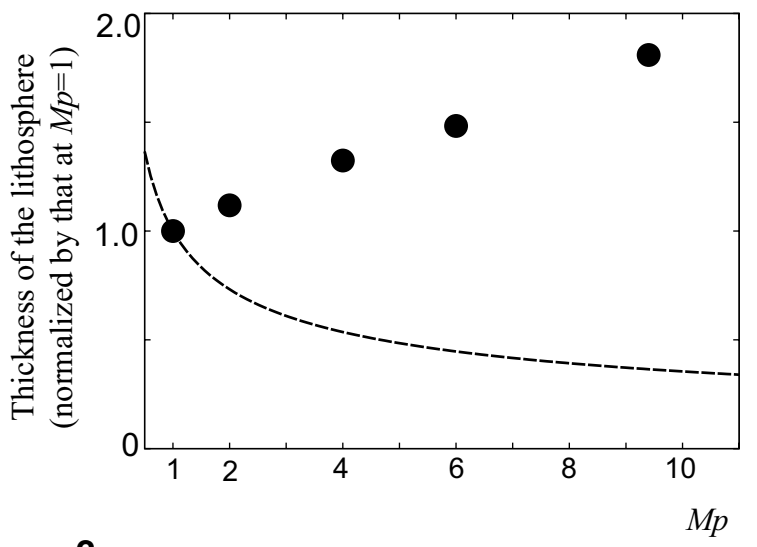

C
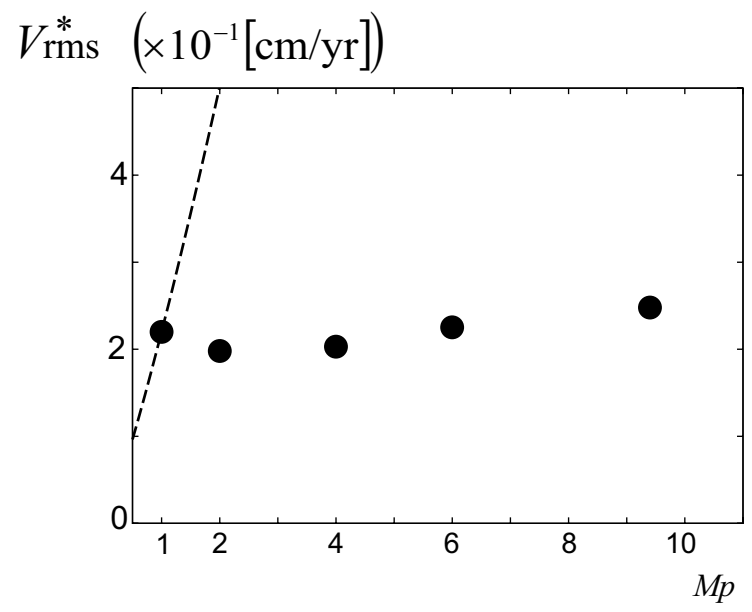

Deschamps and Sotin 2000) where $R a$ depends on $M_{\mathrm{p}}$ as $M_{\mathrm{p}}^{1.34}$, as derived in "Model" section. Thus, the normalized thickness depends on $M_{\mathrm{p}}$ as $L(M p=1) \cdot M p^{-0.346}$ under the Boussinesq approximation, where $L(M p=1)$ is the normalized thickness at $M_{\mathrm{p}}=1$. This dependence is shown by the dotted line in Fig. 3a. The plot shows that the lithosphere becomes substantially thicker, when the
Fig. 3 a The plot of $L$ (the thickness of the lithosphere normalized by the mantle depth) against $M_{\mathrm{p}}$. The dotted line shows the plot that is expected from the Boussinesq approximation $L\left(M_{p}=1\right) \cdot M_{p}^{-0.346}$ where $L\left(M_{p}=1\right)$ is the normalized thickness at $M_{p}=1$ (for details, see the main text). $\mathbf{b}$ The dependence of the thickness of the lithosphere $h=L d^{*}$ on $M_{p}$, where $d^{*}$ is the mantle depth that depends on $M_{p}$ as $d^{*}=d_{\oplus}\left(M_{p}\right)^{0.28}$; the thickness is normalized by its value at $M_{p}=1$. The dashed line shows the dependence suggested earlier from the Boussinesq approximation (Valencia et al., 2007); the thickness depends on $M_{p}$ as $M_{p}^{-0.45}$ in their models. c The dimensional root-mean-square average velocity $V_{\text {rms }}^{*}$ of the convection plotted against $M_{\mathrm{p}}$. The dashed line shows the relationship of $V_{\text {rms }}^{*}\left(M_{p}=1\right) \cdot M_{p}^{1.19}$ suggested in Valencia et al. (2007). (Here, $V_{\text {rms }}^{*}\left(M_{p}=1\right)$ is the value of $V_{\text {rms }}^{*}$ at $M_{p}=1$.)

effect of adiabatic compression is taken into account. (In the figure, the lithosphere at $M_{\mathrm{p}}=1$ is much thicker than that we usually observe for the Earth, because we neglected internal heating and plate motion in our models.)

When converted to dimensional quantity by using $d^{*}=d_{\oplus}\left(M_{\mathrm{p}}\right)^{0.28}$, which is described in "Model" section, the thickness of the lithosphere $\left(=d^{*}\right.$ times the normalized thickness shown in Fig. 3a) increases with increasing $M_{\mathrm{p}}$, as shown in Fig. 3b (again, the thickness is normalized by the value at $M_{\mathrm{p}}=1$ ), despite that a larger $M_{\mathrm{p}}$ implies a thicker mantle, and hence a higher Rayleigh number. The lithosphere becomes about twice thicker, as the planetary mass becomes 9.4 times larger. For comparison, we show the dependence of the dimensional thickness on $M_{\mathrm{p}}$ expected in an earlier parameterized convection model where the Boussinesq approximation is employed (Valencia et al. 2007); here the thickness is expressed as $M_{\mathrm{p}}^{-0.45}$ (the dashed line). When the effects of adiabatic compression are considered, the lithosphere becomes thicker rather than thinner with increasing $M_{\mathrm{p}}$.

We also present a plot of the dimensional root-meansquare velocity of the convection $V_{\text {rms }}^{*}=V_{\text {rms }} \kappa^{*} / d^{*}$ (here $\left.\kappa^{\prime \prime}=10^{-6}\left[\mathrm{~m}^{2} / \mathrm{s}\right]\right)$ against $M_{\mathrm{p}}$. The non-dimensional average velocity $V_{\text {rms }}$ is given as $V_{\text {rms }}=\left\langle\sqrt{\left\langle V_{x}^{2}+V_{z}^{2}\right\rangle_{\text {ave }}}\right\rangle_{\text {time ave' }}$, where $V_{x}$ and $V_{z}$ are the velocity vector component of horizontal and vertical directions, \langle\rangle$_{\text {ave }}$ means spatially averaged, and \langle\rangle$_{\text {time_ave }}$ means time averaged. As shown in the figure, $V_{\mathrm{rms}}^{*}$ is almost independent of $M_{\mathrm{p}}$, when the effect of adiabatic compression is taken into account. Although the non-dimensional $V_{\text {rms }}$ increases by a factor of about 2 as $M_{\mathrm{p}}$ increases from 1 to 9.4 (see the arrows in Fig. 1), this increase in $V_{\text {rms }}$ is canceled out in $V_{\text {rms }}^{*}$ by the increase in $d^{*}$ due to increasing $M_{\mathrm{p}}$. In contrast, in the Boussinesq approximation model (Valencia et al. 2007), the $V_{\text {rms }}$ rapidly increases with increasing $M_{\mathrm{p}}$ as $V_{\mathrm{rms}}^{*}\left(M_{\mathrm{p}}=1\right) \cdot M_{\mathrm{p}}^{1.19}$ where $V_{\mathrm{rms}}^{*}\left(M_{\mathrm{p}}=1\right)$ is the value of $V_{\mathrm{rms}}^{*}$ at $M_{\mathrm{p}}=1$ (see the dashed line in Fig. 3c). 


\section{Discussion}

In our numerical models, the effects of adiabatic compression on thermal convection become substantial in the mantle of super-Earths, when the mass $M_{\mathrm{p}}$ exceeds a threshold value $M_{\mathrm{c}}$ about 4 . Hot ascending plumes become less prominent as $M_{\mathrm{p}}$ increases and cannot directly ascend to the base of the lithosphere in a planet with $M_{\mathrm{p}}$ larger than $M_{\mathrm{c}} \sim 4$. As a consequence, the flows induced by cold descending plumes become the major component of the convective flow at large $M_{\mathrm{p}}$. The thickness of the lithosphere normalized by the mantle depth is also affected by adiabatic compression. The normalized thickness becomes almost independent of $M_{\mathrm{p}}$, and hence the dimensional thickness of the lithosphere increases with increasing $M_{\mathrm{p}}$, as shown in Fig. 3b.

The hot plumes lose their buoyancy as they ascend in the mantle of planets with a large $M_{\mathrm{p}}$, because the temperature contrast between a hot plume and the surrounding mantle $\delta T$ decreases by

$$
\frac{d \delta T}{d z}=-\alpha(z) D i \cdot \delta T,
$$

owing to adiabatic decompression, as the plume ascends. (See Eq. (7).) At $M_{\mathrm{p}}=2(D i=1.49)$, hot plumes ascend to the base of the lithosphere that is at the height $z$ of $\approx$ 0.68 ; the coefficient $\alpha(z) D i$ is about 0.59 there, according to Table 1 and Eq. (5). At $M_{\mathrm{p}}=4(\mathrm{Di}=2.57)$, however, the same value of the coefficient is reached at $z \approx 0.58$, which is well below the base of the lithosphere. At larger $M_{\mathrm{p}}$, the same value of the coefficient $\alpha(z) D i$ is reached even at lower $z$. The lower level of $z$ is the reason why the hot plumes stop ascending before they reach the base of the lithosphere at $M_{\mathrm{p}}$ equal to 4 or greater.

An estimate of the average dissipation number $\overline{D i}=D i \int_{0}^{1} \alpha / \alpha_{0} \mathrm{~d} z$ also leads to the same conclusion. $\overline{D i}$ is $0.44,0.57,0.69,0.74,0.79$ at $M_{\mathrm{p}}=1,2,4,6$, and 9.4, respectively; $\overline{D i}$ at $M_{\mathrm{p}}=4$ is already about $90 \%$ of that at $M_{\mathrm{p}}=9.4$. The rather large value of $\overline{D i}$ at $M_{\mathrm{p}}>4$ is also the reason why the lithosphere becomes thicker (Fig. 3b) and the root-mean-square average velocity does not increase (Fig. 3c) with increasing $M_{\mathrm{p}}$ at $M_{\mathrm{p}}>4$ in spite of the larger $R a$ at larger $M_{\mathrm{p}}$. The stronger effects of adiabatic compression counteract the effects of higher $R a$ at large $M_{\mathrm{p}}$.

The role of adiabatic compression in controlling the thickness of lithosphere is also readily concluded from the comparison with results in our earlier work (Miyagoshi et al. 2015). When both $r$ and $D i$ are fixed, the Nusselt number increases as $R a$ increases (see Figure 8 of Miyagoshi et al. (2015)), indicating the decrease in nondimensional thickness of the lithosphere. (It also shows that its non-dimensional thickness is not determined by only $r$ ). In our present model where both $R a$ and $D i$ increase with $M_{\mathrm{p}}$, in contrast, its non-dimensional thickness does not decrease for given $r$. The difference from the results of Miyagoshi et al. (2015) clearly shows that the almost constant non-dimensional thickness of the lithosphere for the values of $M_{\mathrm{p}}$ employed here (Fig. 3a) is caused by the counteraction between the increase in $R a$ and that in $D i$ with $M_{\mathrm{p}}$.

The thick lithosphere (Fig. 3b) and the inactive convection (Fig. 3c) observed at large $M_{\mathrm{p}}$ imply that it is necessary to revisit the issue of plate tectonics on super-Earths. In the literature, the stress induced in the lithosphere by mantle convection has been estimated and has been compared with the rupture strength of the lithosphere to see if plate tectonics operates in super-Earths (e.g., Valencia et al. 2007; Valencia and O'Connell 2009). In these estimates, the stress is proportional to $V_{\text {rms }} / h^{*}$ where $h$ is the thickness of the lithosphere. Since $V_{\text {rms }}^{*}$ increases and $h^{*}$ decreases with increasing $M_{\mathrm{p}}$ under the Boussinesq approximation, it has been concluded that the stress in the lithosphere exceeds its rupture strength, and that plate tectonics takes place more readily in super-Earths. When the adiabatic compression is taken into account, however, $h^{*}$ increases with increasing $M_{\mathrm{p}}$, and $V_{\mathrm{rms}}^{*}$ is almost independent of $M_{\mathrm{p}}$, as shown in Fig. 3; the figure suggests that the stress decreases as $M_{\mathrm{p}}$ increases, and that plate tectonics may be more difficult to operate in massive super-Earths.

The low activity of hot ascending plumes at large $M_{\mathrm{p}}$ shown in Fig. 1 contains an implication for the volcanism on super-Earths, too. On the Earth, hot plumes are known to cause hot spot volcanism like the one observed at Hawaii and large igneous provinces (LIP) that causes rifting of plates (e.g., Coffin and Eldholm 1994). However, the lowered activity of hot plumes due to the effect of adiabatic compression in our results suggests that such volcanism as the one observed on the Earth is not important in super-Earths, when $M_{\mathrm{p}}$ exceeds around 4.

Of course, we neglected many factors that may affect thermal convection to fix the model presented here. As has been already discussed, the vigor of mantle convection measured by the Nusselt number significantly depends on the surface temperature in massive superEarths Tachinami et al. (2014). The dependence of viscosity on pressure is also important (e.g., Tackley et al., 2013). Besides, we neglected internal heating and highpressure induced phase changes such as the post-perovskite transition. Further numerical studies with these effects are necessary to predict with more confidence the threshold in $M_{\mathrm{p}}$ above which the effects of adiabatic compression becomes important.

In summary, our numerical models suggest that the strong effects of adiabatic compression make both plate tectonics and volcanism caused by hot ascending plumes 
difficult to take place in super-Earths with $M_{\mathrm{p}}$ larger than about 4 under our model settings. To confirm this conclusion, further numerical experiments are necessary in the future, taking account of the complicated rheology of mantle materials (e.g., pressure- or stress-dependent viscosity), internal heating and the long-lasting effects of the initial condition on the thermal state of the mantle (Miyagoshi et al. 2017).

\section{Authors' contributions}

TM performed numerical simulations, analysis of simulation data, and prepared the manuscript. MK developed the numerical simulation code ACUTEMan and prepared the manuscript. MO prepared the manuscript. All authors discussed numerical simulation results. All authors read and approved the final manuscript.

\section{Author details}

1 Department of Deep Earth Structure and Dynamics Research, Japan Agency for Marine-Earth Science and Technology, 3173-25 Showa-machi, Kanazawa-ku, Yokohama, Kanagawa 236-0001, Japan. ${ }^{2}$ Geodynamics Research Center, Ehime University, 2-5 Bunkyo-cho, Matsuyama, Ehime 790-8577, Japan. ${ }^{3}$ Department of Earth Sciences and Astronomy, University of Tokyo at Komaba, 3-8-1 Komaba, Meguro, Tokyo 153-8902, Japan.

\section{Acknowledgements}

The authors thank two anonymous reviewers for their helpful comments. Numerical simulations were performed on the Earth Simulator and the supercomputer system at Japan Agency for Marine-Earth Science and Technology.

\section{Competing interests}

The authors declare that they have no competing interests.

\section{Availability of data and materials}

Data supporting the figures in this article are available upon request to the authors.

\section{Consent for publication}

Not applicable.

\section{Ethics approval and consent to participate}

Not applicable.

\section{Funding}

This work was supported by JSPS KAKENHI Grant Numbers JP25287110, JP15H05834, a joint research program at Geodynamics Research Center, Ehime University, and "Exploratory Challenge on Post-K computer" (Elucidation of the Birth of Exoplanets [Second Earth] and the Environmental Variations of Planets in the Solar System).

\section{Publisher's Note}

Springer Nature remains neutral with regard to jurisdictional claims in published maps and institutional affiliations.

Received: 16 July 2018 Accepted: 11 December 2018 Published online: 19 December 2018

\section{References}

Borucki WJ, Koch DG, Basri G et al (2011) Characteristics of planetary candidates observed by Kepler. II. analysis of the first four months of data. Astrophys J 736:19. https://doi.org/10.1088/0004-637x/736/1/19

Coffin MF, Eldholm O (1994) Large igneous provinces: crustal structure, dimensions, and external consequences. Rev Geophys 32:1-36
Davies GF (2011) Mantle convection for Geologists. Cambridge Univ. Press, U. K.

Deschamps F, Sotin C (2000) Inversion of two-dimensional numerical convection experiments for a fluid with a strongly temperature-dependent viscosity. Geophys J Int 143:204-218

Foley BJ, Bercovici D, Landuyt W (2012) The conditions for plate tectonics on super-Earths: inferences from convection models with damage. Earth Planet Sci Lett 331-332:281-290. https://doi.org/10.1016/j. epsl.2012.03.028

Kameyama M (2005) ACuTEMan: a multigrid-based mantle convection simulation code and its optimization to the Earth Simulator. Journal of the Earth Simulator 4:2-10

Kameyama M, Yamamoto M (2018) Numerical experiments on thermal convection of highly compressible fluids with variable viscosity and thermal conductivity: implications for mantle convection of super-Earths. Phys Earth Planet Inter 274:23-36. https://doi.org/10.1016/j.pepi.2017.11.001

Kameyama M, Kageyama A, Sato T (2005) Multigrid iterative algorithm using pseudo-compressibility for three-dimensional mantle convection with strongly variable viscosity. J Comput Phys 206:162-181. https://doi. org/10.1016/j.jcp.2004.11.030

Karato S (2011) Rheological structure of the mantle of a super-Earth: some insights from mineral physics. Icarus 212:14-23. https://doi.org/10.1016/j. icarus.2010.12.005

Lenardic A, Crowley JW (2012) On the notion of well-defined tectonic regimes for terrestrial planets in this solar system and others. Astrophys J 755:132. https://doi.org/10.1088/0004-637X/755/2/132

Miyagoshi T, Tachinami C, Kameyama M, Ogawa M (2014) On the vigor of mantle convection in super-Earths. Astrophys J Lett 780:L8. https://doi. org/10.1088/2041-8205/780/1/L8

Miyagoshi T, Kameyama M, Ogawa M (2015) Thermal convection and the convective regime diagram in super-Earths. I Geophys Res Planets 120:1267-1278. https://doi.org/10.1002/2015JE004793

Miyagoshi T, Kameyama M, Ogawa M (2017) Extremely long transition phase of thermal convection in the mantle of massive super-Earths. Earth Planets Space 69:46. https://doi.org/10.1186/s40623-017-0630-6

O'Neill C, Lenardic A (2007) Geological consequences of super-sized Earths. Geophys Res Lett 34:L19204. https://doi.org/10.1029/2007GL030598

Richards MA, Duncan RA, Courtillot VE (1989) Flood basalts and hot-spot tracks: plume heads and tails. Science 246:103-107. https://doi. org/10.1126/science.246.4926.103

Schubert G, Turcotte DL, Olson P (2001) Mantle convection in the earth and planets. Cambridge Univ. Press, Cambridge

Stamenković $\vee$, Breuer D (2014) The tectonic mode of rocky planets: part 1driving factors, models \& parameters. Icarus 234:174-193. https://doi. org/10.1016/j.icarus.2014.01.042

Stein C, Lowman JP, Hansen U (2013) The influence of mantle internal heating on lithospheric mobility: implications for super-Earths. Earth Planet Sci Lett 361:448-459. https://doi.org/10.1016/j.epsl.2012.11.011

Tachinami C, Senshu H, Ida S (2011) Thermal evolution and lifetime of intrinsic magnetic fields of super-earths in habitable zones. Astrophys J 726:70. https://doi.org/10.1088/0004-637X/726/2/70

Tachinami C, Ogawa M, Kameyama M (2014) Thermal convection of compressible fluid in the mantle of super-Earths. Icarus 231:377-384. https://doi. org/10.1016/j.icarus.2013.12.022

Tackley PJ, Ammann M, Brodholt JP, Dobson DP, Valencia D (2013) Mantle dynamics in super-Earths: post-perovskite rheology and self-regulation of viscosity. Icarus 225:50-61. https://doi.org/10.1016/j.icarus.2013.03.013

Valencia D, O'Connell RJ (2009) Convection scaling and subduction on Earth and super-Earths. Earth Planet Sci Lett 286:492-502. https://doi. org/10.1016/j.epsl.2009.07.015

Valencia D, O'Connell RJ, Sasselov DD (2006) Internal structure of massive terrestrial planets. Icarus 181:545-554. https://doi.org/10.1016/j.icarus.2005.11.021

Valencia D, O'Connell RJ, Sasselov DD (2007) Inevitability of plate tectonics on super-earths. Astrophys J Lett 670:L45-L48. https://doi. org/10.1086/524012

van Heck HJ, Tackley PJ (2011) Plate tectonics on super-Earths: equally or more likely than on Earth. Earth Planet Sci Lett 310:252-261. https://doi. org/10.1016/j.epsl.2011.07.029 